\title{
Shape memory alloy based motor
}

\author{
S V SHARMA ${ }^{1}$, M M NAYAK $^{1}$ and N S DINESH ${ }^{2}$ \\ ${ }^{1}$ Indian Space Research Organisation, Bangalore 560094 \\ ${ }^{2}$ Indian Institute of Science, Centre for Electronics Design and Technology, \\ Bangalore 560012 \\ e-mail: svsharma@isac.gov.in; mmnayak@sclchd.co.in; dinesh@cedt.iisc.ernet.in
}

\begin{abstract}
Design and characterization of a new shape memory alloy wire based Poly Phase Motor has been reported in this paper. The motor can be used either in stepping mode or in servo mode of operation. Each phase of the motor consists of an SMA wire with a spring in series. The principle of operation of the poly phase motor is presented. The motor resembles a stepper motor in its functioning though the actuation principles are different and hence has been characterized similar to a stepper motor. The motor can be actuated in either direction with different phase sequencing methods, which are presented in this work. The motor is modelled and simulated and the results of simulations and experiments are presented. The experimental model of the motor is of dimension $150 \mathrm{~mm}$ square, $20 \mathrm{~mm}$ thick and uses SMA wire of $0.4 \mathrm{~mm}$ diameter and $125 \mathrm{~mm}$ of length in each phase.
\end{abstract}

Keywords. Shape Memory Alloy (SMA); poly phase; rotary actuator; torque; ripple.

\section{Introduction}

Shape memory refers to the ability of certain materials to 'remember' a 'shape or size' at a specified temperature Duerig et al (1990) and Mellor (1989). Due to their unique life like behaviour, they are also called intelligent or smart material. SMA is malleable at low temperature, and can be deformed to any shape by applying a small force. It can be trained (Duerig et al 1990) to remember a specific shape at a high temperature. Even if deformed at low temperature, when SMA is heated to a predetermined transformation temperature it assumes the trained shape. In this high temperature state it needs large force to deform. The low temperature state is called Martensite phase and high temperature state is called Austenite phase. The shape memory effect occurs as a result of a change in the atomic crystal structure (Duerig et al 1990) of the alloy. Unlike conventional materials, which show only, limited effect on stress-strain behaviour (Duerig et al 1990; Mellor 1989), SMA shows marked temperature dependence, because of reversible austenite to martensite transformation. The underlying phenomenon of the shape memory effect is quite complex. The detailed properties of the dynamics of shape memory alloys vary widely with their metallurgy content, fabrication process, training techniques, aging, and ambient conditions. Shape memory materials are 
available in variety of sizes and shapes such as wires, rods, springs and strips. SMA can be classified mainly in to two categories namely, One way shape memory and Two way shape memory. The One way shape memory remembers high temperature state, and a small external force is required to bring it back to original shape when cooled. Two way shape memory can remember both high temperature and low temperature states. One way shape memory has the advantage of higher strain for a given length compared to Two way shape memory.

Using SMA materials different types of actuators have been made. SMA actuators can be broadly classified into Limited Motion Type and Unlimited Motion Type. Under limited motion type both linear and rotary type actuators are presented in literature. Under unlimited motion type actuators only one type of motor is reported in literature Dominiek \& Van Brussel (1998). The motor reported uses SMA springs for actuation.

Comparison is made in literature Otsuka \& Wayman (1989) and Dominiek \& Van Brussel (1998) for the actuators designed using the SMA element in tension mode (wire actuator), torsion mode (spring actuator) and bending mode (strip actuator). While the SMA actuator designed using SMA strip has an energy density (Dominiek \& Van Brussel 1998) of 4.6 J/kg compared to SMA actuator designed using SMA wire has an energy density of $466 \mathrm{~J} / \mathrm{Kg}$. It is clear that the SMA used in tension mode has 100 times better energy density compared to SMA used in bending mode. Therefore, in this paper, we have attempted to build a balanced Poly Phase Motor, which uses SMA wire in series with a spring in the tension mode. The motor generates more torque compared to the motor designed with spring or strip type SMA actuator for the same volume of SMA material used. It is observed in literature (Kuribayashi 1989a,b) that SMA spring or strip generates force as a non-linear function of its deformed length. The actuation used in the present work isolates the function of spring from SMA actuation and hence generates a constant effective force throughout its operating range. The motor presented, works in stepping mode (Kuo 1979) generating incremental motion and servo mode generating continuous motion. The SMA wire based motor presented has better dynamic response compared to the motors that use SMA strips or springs Kuribayashi 1989a; 1989 b.

Looking at the energy efficiency of an SMA actuator, it can never have a greater efficiency than a Carnot cycle between heating and cooling temperatures. Because of this, an SMA actuator will never compete with classical electric actuators. But the SMA has very high power to weight ratio and power to volume ratio compared to the conventional electric actuators and hydraulic actuators (Dominiek \& Van Brussel 1998). There are several applications where the energy efficiency is not of concern but a large power has to be generated with in a compact size. Therefore, SMA has found wide usage in space application due to its compact size, non magnetic property, smooth, jerk free operation and insensitivity to space radiations namely, electrons, protons and heavy ions. SMA also has found applications in toys, medical implants, fashion designs, aerospace, etc.

\section{Operation of poly phase SMA motor}

\subsection{Principle of one way shape memory actuation}

Figure 1 shows the principle of One-way shape memory actuation. Nickel and Titanium metal in a near equal atomic ratio is used to make an SMA alloy NiTi. The NiTi alloy is trained to remember a short length at high temperature by heat treatment (Duerig et al 1990). When in cold state (Martensite) the SMA is malleable and can be stretched to a longer length with a small force. Normally in SMA actuators a biasing force is applied during the cold condition 


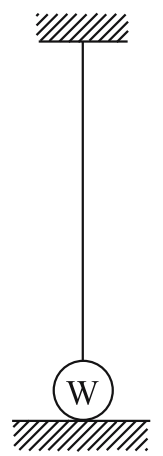

Low temperature
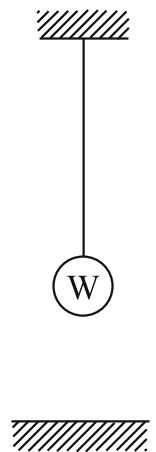

High

temperature

Figure 1. One way SMA actuation.

to stretch the SMA wire to a designed length and this is known as pre-loading. Pre-loading is usually done by using a spring in series with the SMA wire or a weight to generate force by gravity (W in figure 1). When the SMA is heated to high temperature (Austenite), SMA shrinks to the trained length. While shrinking, the SMA becomes hard, and can pull much larger force than what is required to stretch SMA at low temperature. The proposed poly phase motor is designed using one way SMA wires.

\subsection{Principle of operation of poly phase SMA motor}

The principle of functioning of the proposed Poly Phase Motor is given in figure 2. Three identical SMA wires in series with identical springs which form the three phases of the motor are fixed to a cam and the other ends of the springs are connected to fixed points Anchor1, 2 and 3 spaced $120^{\circ}$ apart. If the cam is rotated by applying external force, the springs expand or contract to accommodate change in distance between the fixed points and the cam. The

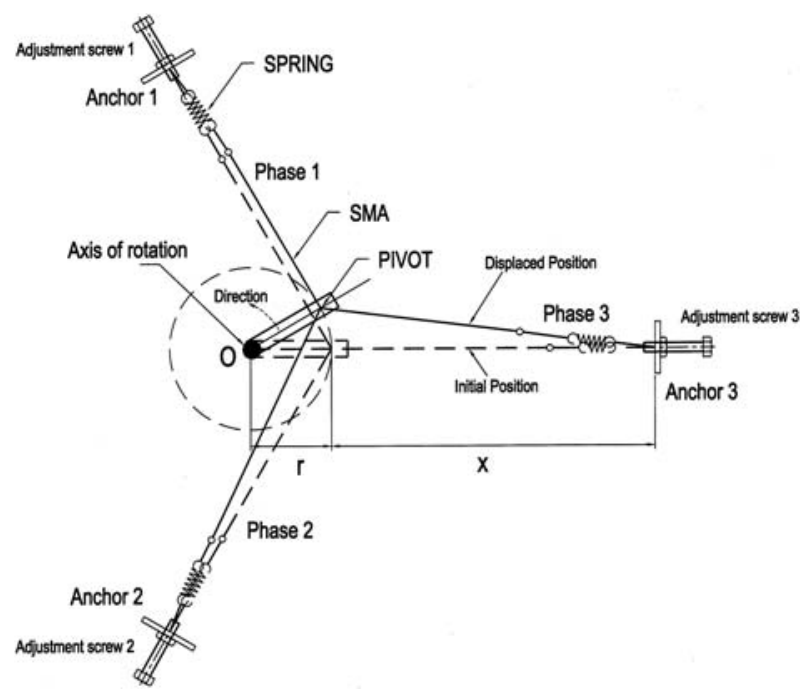

Figure 2. Principle of poly phase SMA motor. 


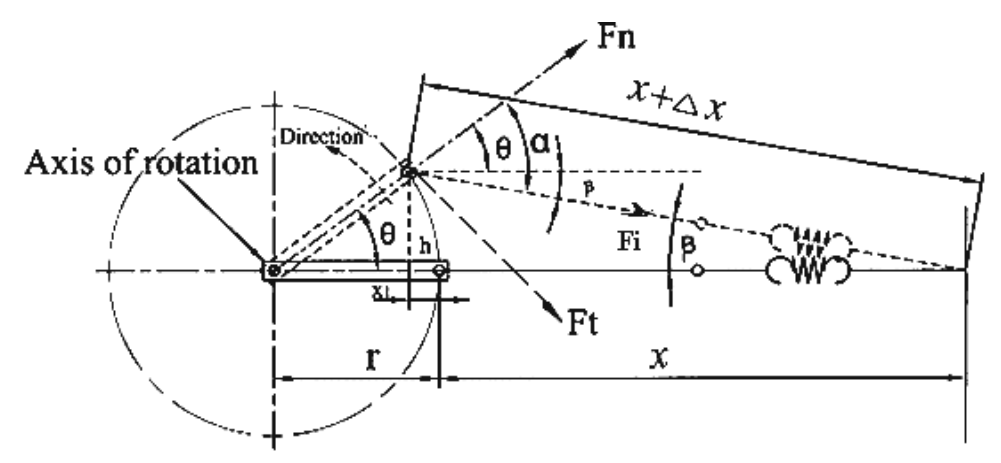

Figure 3. Geometric topology of a phase.

tensions in the springs and the wire arrangement are properly balanced so that the resultant force due to the three Phases on the cam is minimized. Therefore, in the static condition when all Phases are at the same temperature, cam is nearly balanced and stable in all positions and it needs very little external force to rotate it. The motor can be rotated by heating different SMA wires one after the other in a particular sequence. Let the initial position of the cam be as indicated in figure 2. To rotate the motor in the anticlockwise direction, Phase1 SMA is actuated by heating. This is done by passing current through that SMA wire, which in turn generates heat proportional to the resistance of SMA wire and square time the current. As the SMA wire heats up sufficiently to generate a compressive force in it, it pulls the cam towards Anchor1. The motor shaft rotates and when it reaches the point where the cam, SMA wire of Phase 1 and the corresponding spring are all in the same line. At this position there will not be any torque acting on the cam and it settles at $120^{\circ}$ with respect to the initial position. Now the same process is repeated with Phase2 SMA Wire with Phase1 SMA is switched OFF. As the Phase 1 is switched OFF and it cools to the ambient due to forced air-cooling it generates no extra torque. At the same time Phase 2 is switched $\mathrm{ON}$ and it pulls the cam toward Anchor2 and the cam finally settles at $240^{\circ}$ with reference to the initial position. Now by switching 'OFF' Phase 2 and switching 'ON' Phase 3 we can move the cam to the initial position. This sequence of actuation (Phase1 - Phase2 - Phase3) is repeated for continuous rotation of the cam in the anti clockwise direction. To rotate the cam in the clockwise direction, the sequence of actuation of the phases is in the order Phase2 - Phase1 - Phase3.

\subsection{Torque equation}

Figure 3 shows the geometric topology of one phase of the motor at an arbitrary instant. Based on the geometric relations, expression for the torque, force and the variation of the spring length due to the applied external force are derived as detailed below.

$$
T=\sum_{i=1}^{n} r * F i * \sin \alpha i,
$$

where $i$ is the Phase number, $F_{i}$ is the force generated in the $i^{\text {th }}$ Phase.

$$
\beta i=\tan ^{-1}\left[\frac{r \sin \left\{\theta+\frac{2 \pi(n-i)}{n}\right\}}{x+r\left\{1-\cos \left\{\theta+\frac{2 \pi(n-i)}{n}\right\}\right\}}\right]
$$




$$
\begin{aligned}
& \alpha i=\theta+\beta i-\frac{2 \pi}{n} i \\
& F i=K * \Delta x i \\
& \Delta x i=\sqrt{\left[x+r\left\{1-\cos \left(\theta+\frac{2 \pi(n-i)}{n}\right)\right\}\right]^{2}+\left[r \sin \left(\theta+\frac{2 \pi(n-i)}{n}\right]^{2}\right.}-x
\end{aligned}
$$

$K=$ Compliance of spring,$\quad x=$ Initial length of the Phase,$\quad \theta=$ Cam angle,$\quad r=$ Cam radius, $n=$ Number of phases.

\subsection{Static torque/holding torque}

It is defined as the torque generated by the motor versus its displacement for a constant temperature in a phase or phases of the SMA motor. This torque acts in a direction to force the rotor back to and hold it in the zero torque position. The holding torque curve completely determines the instantaneous torque of the motor under all possible static conditions of excitations and rotor position. The static torque curve of the motor for Single-Phase-On and a given temperature is determined by keeping one of the phases actuated at the required temperature by passing current through the SMA, and displacing the rotor to different positions by applying external torque. Similar torque curves can be plotted with different temperatures for single or many phases at the same time and these curves indicate the torque the motor can generate when it is actuated at various displacement angles. Figure 4 shows the simulated and experimental Single-Phase-On static torque curves of the SMA Motor for different phases. Curves 1, 2 and 3 of figure 4 correspond to Phase 1, 2 and 3, respectively.

\section{Operating the actuator by phase sequencing}

\subsection{One-phase-on sequencing (OS)}

A phase is termed switched On or actuated, by passing current through the SMA of the Phase. From the figure 4 we can see that static torque curve for each phase ON, has a stable

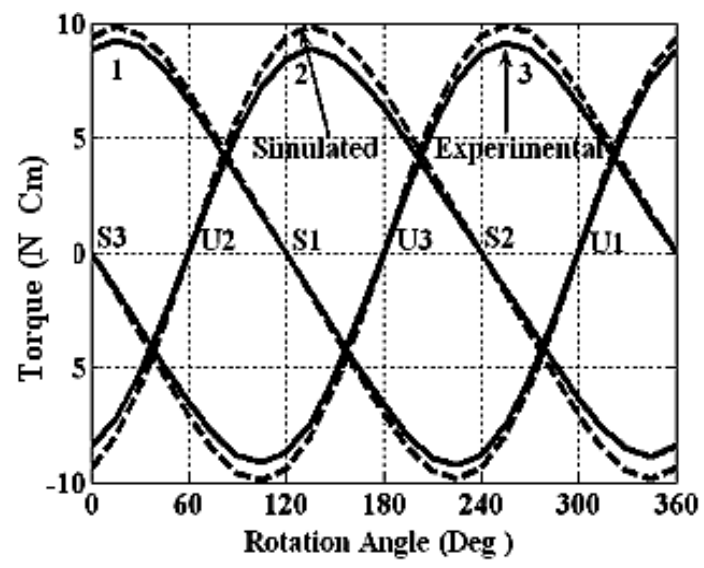

Figure 4. Simulated and experimental static torque curves for the three-phase motor. 


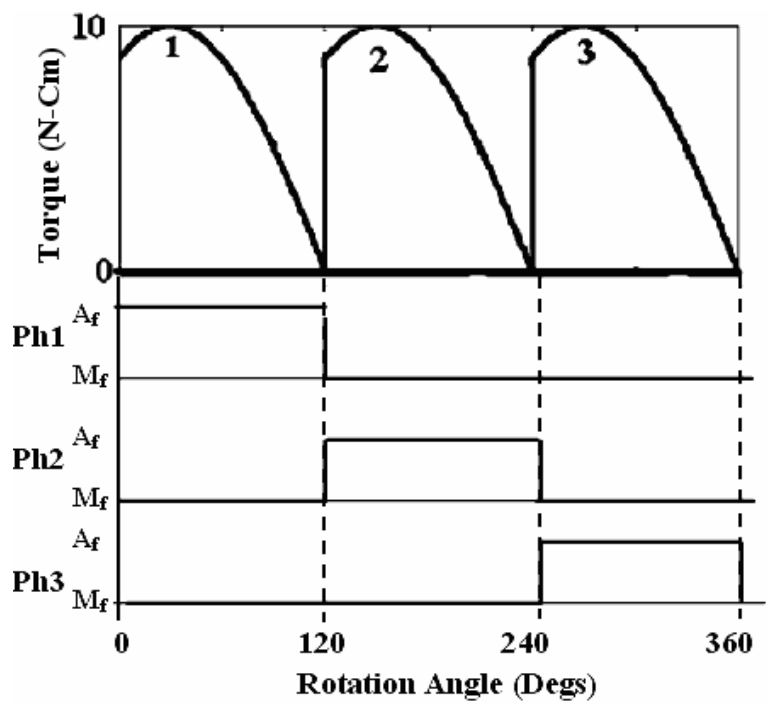

Figure 5. Ideal torque for OS and phase sequencing.

equilibrium position indicated by $\mathrm{S} 1, \mathrm{~S} 2$, and $\mathrm{S} 3$. Let us assume that initially Phase 3 is ON and the rotor will assume the position S3. Now Phase3 is switched OFF (allowed to cool to austenite start temperature) and Phase 1 is switched ON, by passing current through Phase 1 SMA. The motor generates 0.8 times the positive peak torque $T_{\text {peak }}$ at $0^{\circ}$. The motor starts moving anticlockwise to the stable equilibrium point at $120^{\circ}, \mathrm{S} 1$, corresponding to Phase 1 . Now, Phase1 is switched OFF and allowed to cool and Phase 2 is switched ON, by passing current through Phase 2 SMA, to move the motor to the detent position S2 at $240^{\circ}$. On reaching S2, Phase 2 is switched OFF and Phase 3 is switched ON, by passing current through Phase 3 SMA, to move the motor to $\mathrm{S} 3$ at $360^{\circ}$. This sequence of actuation viz. Phase $1 \rightarrow$ Phase $2 \rightarrow$ Phase 3 is repeated for continuous rotation of the motor in the anticlockwise direction and for each sequence the motor moves by a step of $120^{\circ}$. In this type of actuation we are switching one Phase ON at a time and hence can be called One-Phase-On Sequencing (OS). The step angle covered by the motor can be defined as the 'natural step angle'-the angle moved by the motor for every sequence in OS. It is clear from above illustration that the natural step angle for a 3 Phase motor is $120^{\circ}$. To rotate the motor in the clockwise direction, the sequence of actuation of the Phases is reversed. Figure 5 shows the simulated torque and the phase sequencing of the motor during the OS.

From the figure it is clear that the motor generates torque varying from zero to the maximum as it moves from one detent position to the other. There is a large torque ripple. We have not considered the dynamics of torque generation by the motor in figure 5. Figure 6 shows the simulated torque generated by the motor for OS considering the time constants associated with the thermal component and the mechanical load. We see that the Phase switched off is still hot and generates negative torque and as a result there is a small kink in the static torque curve. Figure 9 shows the response of the motor for the three consecutive steps for the OS.

\subsection{Improved one-phase-on sequencing (IOS)}

In figure 4 we observe that Phase 2 generates positive torque even before reaching S1. From figure it is clear that by sequencing the Phases at the points where the static torque curves 


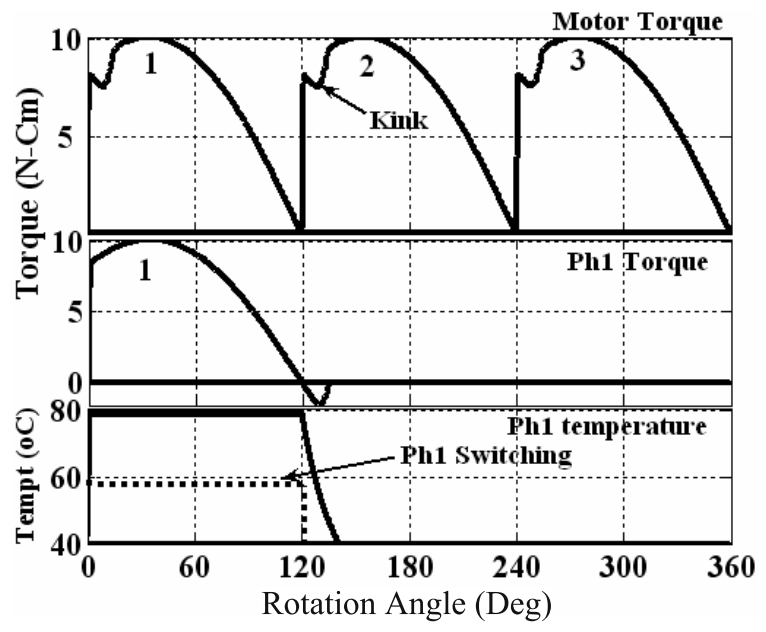

Figure 6. Torque for one-phase-on sequencing.

crossover we can achieve better torque characteristics of the motor i.e. Improved One-PhaseOn-Sequencing (IOS). The torque crossover points are dependent on the construction of the motor. Figure 7 shows the simulated static torque for IOS and the phase sequencing without considering the thermal time constant. In order to implement this scheme of sequencing we need to find the position of torque crossover by means of a position feedback. Figure 8 shows the torque generated during IOS considering the time constants of the thermal and the mechanical elements in the system.

OS method is simple to implement and requires lesser hardware and consumes lesser energy for its operation compared to IOS. Figure 9 shows the improvement in response of IOS over OS. IOS generates better torque and dynamic response compared to OS, but needs the positional feedback which increases the complexity of the system and hence the increased cost.

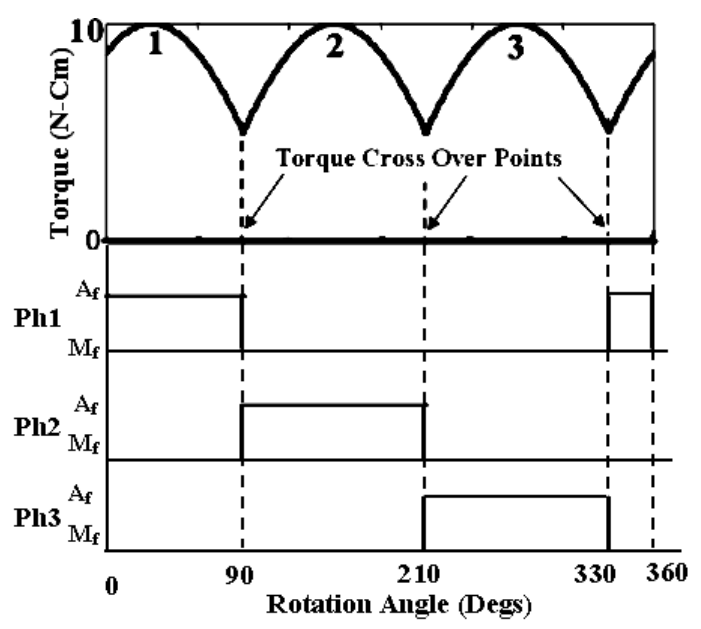

Figure 7. Ideal torque for improved OS and phase sequencing. 


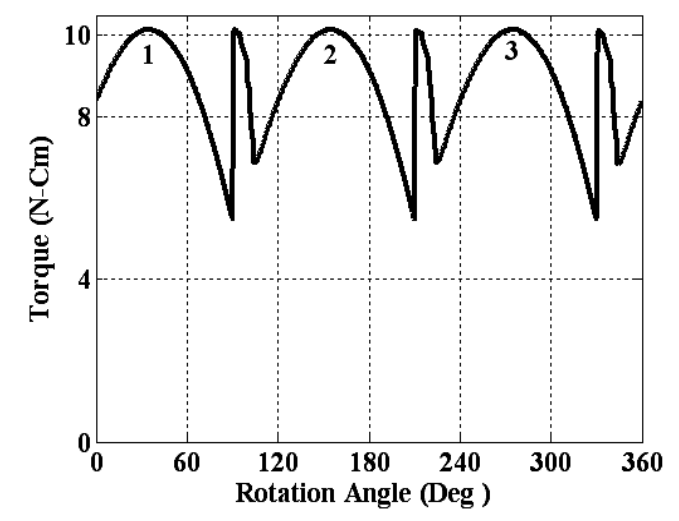

Figure 8. Torque for improved IOS.

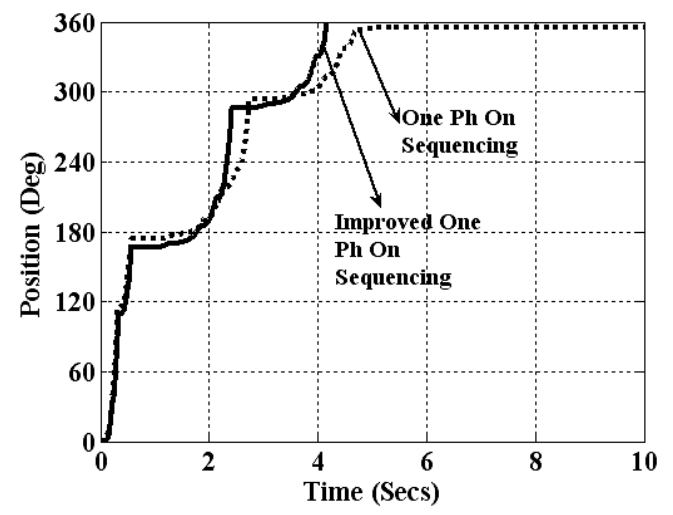

Figure 9. Experimental step response.

\subsection{Two-phase-on sequencing (TOS)}

Figure 10 shows the static torque characteristic of the motor with two Phases kept On, by passing current through them. The characteristic curve resembles that of OS but only is physically shifted with respect to the other. The stable equilibrium has shifted by $60^{\circ}$. Therefore it is pos-

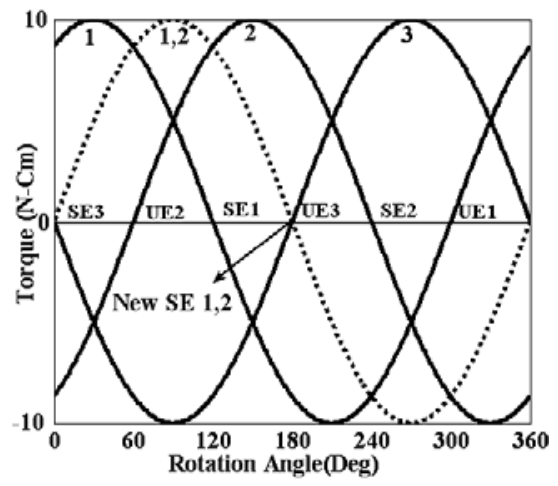

Figure 10. Static torque for two-phase-on sequencing. 


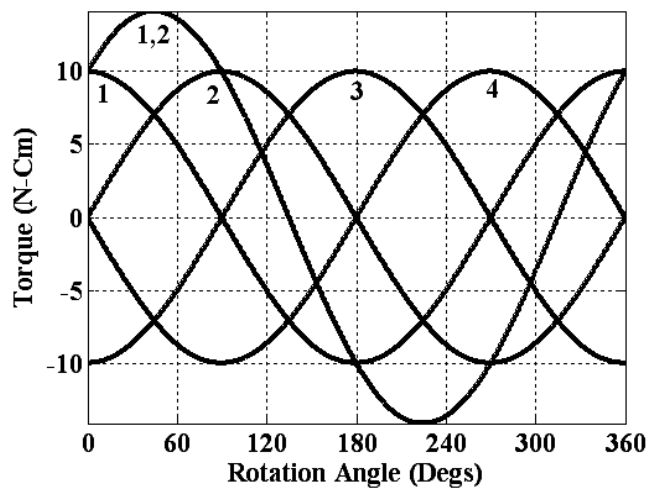

Figure 11. Static torque for TOS for 4 phase motor.

sible to sequence the motor with Two-Phase-On at a time and rotate the motor. The sequence for anticlockwise rotation is: Phase $1+$ Phase $2 \rightarrow$ Phase $2+$ Phase $3 \rightarrow$ Phase $3+$ Phase 1 . The step angle remains same as that of OS. The Two-Phase-On Sequencing (TOS) has no advantage for three Phase Motor in terms of the motor torque. For motors more than three Phases we will get better torque generated when TOS scheme is used compared to OS. For example, in figure 11 we have static torque characteristics for a four Phase Motor with TOS and OS schemes. From the figure it is seen that Two-Phase-On generates more torque. Also the slope at the detent position is more for TOS compared to that with OS resulting in lesser steady state position error.

\subsection{Half step sequencing}

The Half Step Sequencing can be achieved by combining the OS and TOS Sequencing methods. Figure 12 shows the simulation for the torque generated by the motor for Half Step Sequencing.

In Half Step Sequencing, Phases are switched On in the sequence: Phase $1 \rightarrow$ Phase $1+$ Phase $2 \rightarrow$ Phase $2 \rightarrow$ Phase $2+$ Phase $3 \rightarrow$ Phase $3 \rightarrow$ Phase 3 + Phase 1 . For every sequence, the motor moves by half of its natural step. Figure 12 shows the simulation for the torque generated by the motor for Half Step Sequencing and letting the motor to settle down at

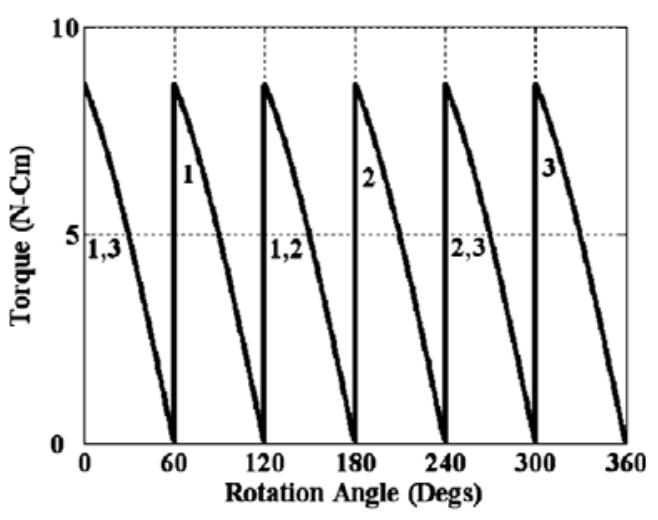

Figure 12. Half step sequencing. 


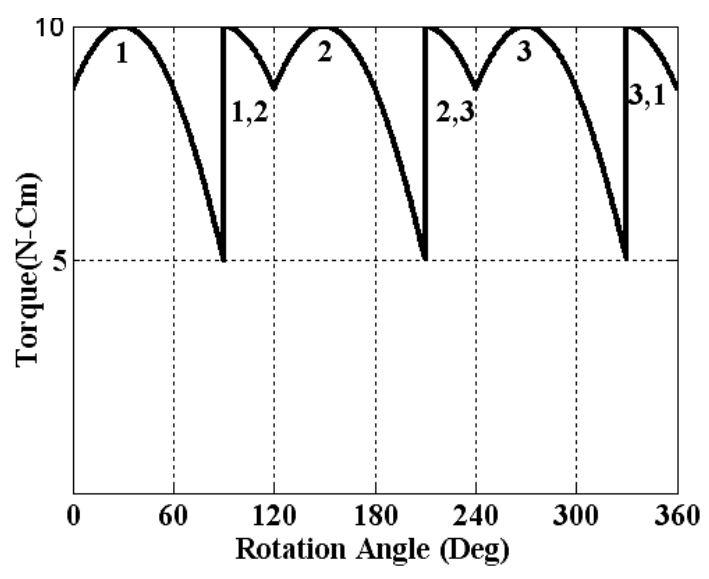

Figure 13. Half step cross over sequencing.

every half step and neglecting the effect of time constants of the thermal and the mechanical elements. For a 3 Phase Motor half step angle is $60^{\circ}$.

For continuous running of the motor, it can be Half Step Sequenced for better torque by sequencing at the torque crossover points. figure 13 shows simulated torque curve for such sequencing with thermal and mechanical time constants neglected.

\subsection{Poly phase sequencing}

In the Poly Phase Sequencing only phases generating positive torque at the given position will be switched $\mathrm{ON}$ for anticlockwise rotation. The mathematical expression for the torque generated by the motor due to sequencing is given below.

$$
T_{\text {poly }}=\sum_{i=1}^{n} \sum_{\theta=(1-i) * 2 \pi / n}^{\theta=\pi+(1-i) * 2 \pi / n} F i * r * \sin \alpha i,
$$

where $\alpha i$ and $F i$ are defined in expression (3) and (4). Poly Phase Sequencing method requires a positional feedback. Based on the construction of the motor, algorithm is generated for

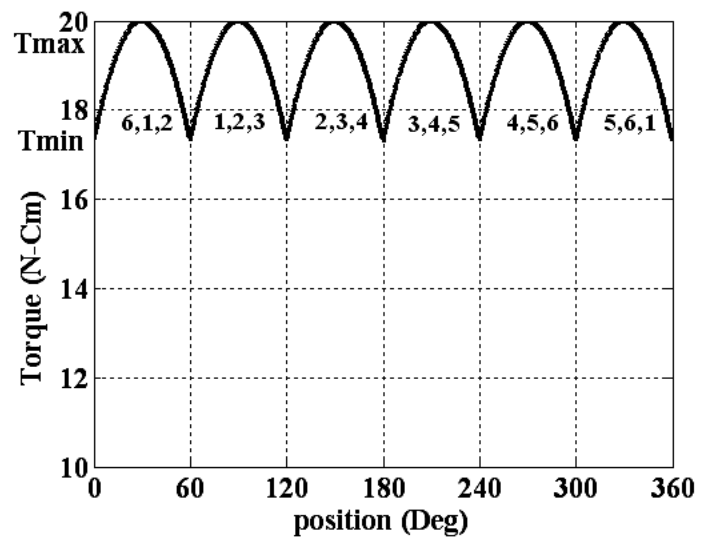

Figure 14. Poly Phase sequencing for 6 phase motor. 


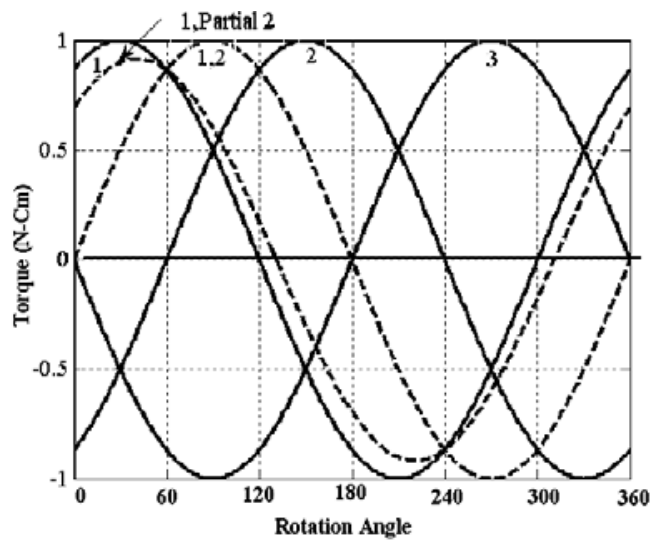

Figure 14a. Static torque of 3 phase motor, Phase $1+$ Phase2 ON, Phase1 ON + Phase2 partial.

sequencing the phases with respect to position of the motor. An electronic controller is used which, switches on the corresponding phases based on the positional feedback. This method of sequencing consumes higher energy compared to the other methods discussed above and also more complex compared to the earlier method of sequencing. In the case motors up to four phases sequencing is same as TOS. But for motors with higher number of phases, Poly Phase Sequencing requires switching of more than two phases at a time. Figure 14 shows the motor torque for a six-phase motor with sequencing.

\subsection{Micro stepping}

We have seen that when Phase1 is kept ON and later Phase1 and Phase 2 are switched ON, there is a half step motion by the motor. Now, instead of switching ON the second Phase with full temperature, if Phase 2 is switched ON partially and Phase1 with full temperature, the motor moves by less than half step depending upon the temperature of Phase 2 . This way of moving the motor by fraction of its natural step angle is shown in figure $14 \mathrm{a}$ and is called micro stepping.

\section{Modelling of the motor}

\subsection{Model of a phase}

The model of one phase of the motor is shown in figure 15. Current flows through the selected phase based on the PID controller O/P. The current to temperature block provides relationship

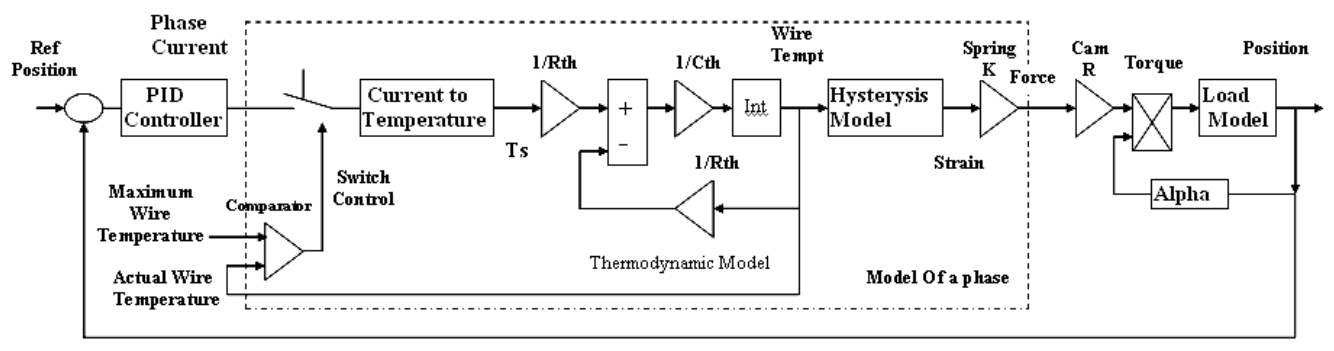

Figure 15. Simulink model of a phase. 


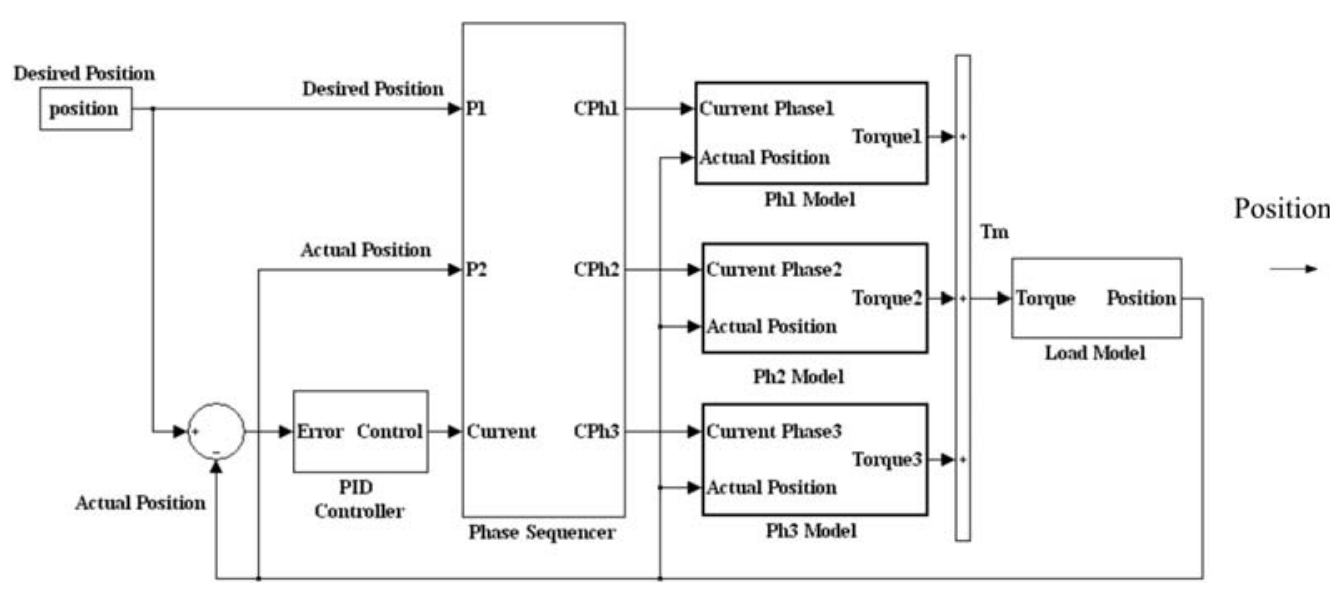

Figure 16. Model of 3-phase SMA motor.

between steady state temperature and the current through the phase. Environmental loss is accounted by thermodynamic model of SMA. Based on the wire temperature, strain (displacement) of the SMA is computed using Hysterysis model. The force generated in a phase is product of compliance of the spring and the displacement of SMA. From the force, torque is found by using the geometric relationship. An ON/OFF current control loop is used to limit the SMA wire temperature to safe value.

\subsection{Motor model}

Figure 16 shows the schematic model of SMA Motor. Phase sequencer is an algorithm to select the appropriate phase for actuation depending on the desired position, actual position and the $\mathrm{PID}$ controller output. $\mathrm{CPh} 1, \mathrm{CPh} 2$ and $\mathrm{CPh} 3$ are the currents through the $\mathrm{Ph} 1, \mathrm{Ph} 2$ and $\mathrm{Ph} 3$ respectively. Each phase develops torque based on controller current. The torques developed by individual phases is vectorially added to get the motor torque.

\section{Experiments}

Figure 17 shows the Three Phase Motor constructed by the authors. The motor is constructed with NiTi (SMA) wire that shrinks on heating. The diameter of the SMA wire is $0.35 \mathrm{~mm}$ and length is $120 \mathrm{~mm}$ in each Phase. The motor is of dimension $150 \mathrm{~mm}$ Square, $20 \mathrm{~mm}$ thick without considering the optical encoder, load and other fixtures used for testing of the motor. It must be noted that the motor is built to demonstrate the concept and there is scope for reducing the size of the motor considerably by appropriate mechanical design. The motor develops peak torque of $10 \mathrm{~kg}$-mm by using spring of $0.1 \mathrm{Kg} / \mathrm{mm}$ compliance and peak torque of $20 \mathrm{Kg} / \mathrm{mm}$ by using spring of $0.2 \mathrm{Kg} / \mathrm{mm}$ compliance. The schematic block diagram of the electronic hardware for controlling the motor phases for positioning and rotating the motor is shown in figure 18 . The temperature of each selected phase is monitored using a thermocouple mounted on each phase SMA and accordingly the pulse width of the drive is controlled to keep the temperature with in the desired limits. The maximum current through each phase will be $1 \mathrm{Amp}$ DC. The motor rotates at a maximum speed of about 10 revolutions per minute with forced air-cooling. The position of the motor is measured using an optical encoder with 


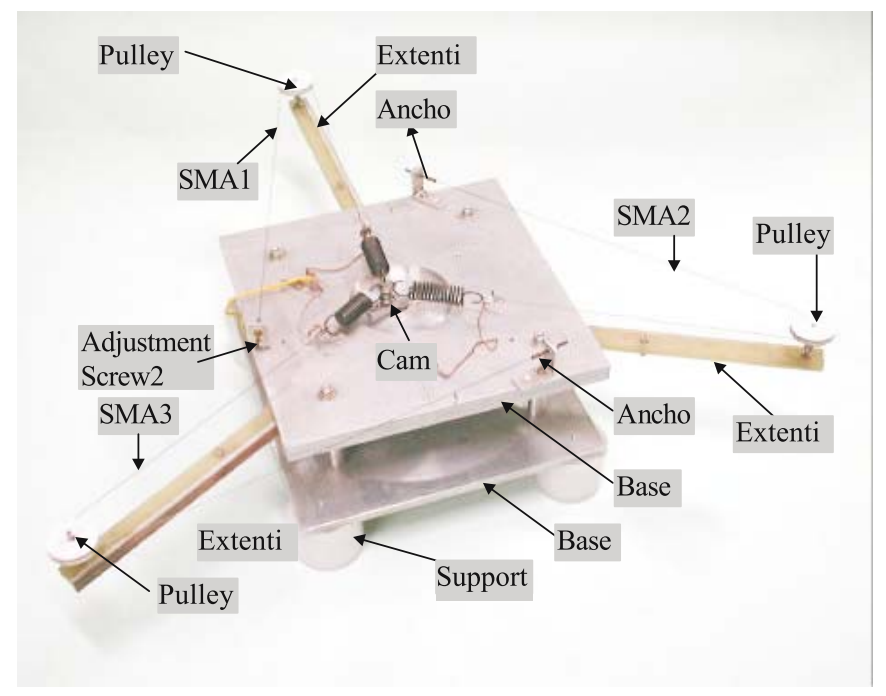

Figure 17. Photograph of laboratory model of the 3-phase SMA motor.

a resolution of $0.025^{\circ}$, and the position information is used for sequencing operation. The response of the motor for IOS for five consecutive full rotations is presented in figure 19. The experimental result shows the effect of thermal and mechanical time constants of the motor elements.

\section{Summary}

This paper dealt with construction of a new type of SMA based Poly Phase Motor. Relationship between the motor torque and the SMA strain was presented. While characterizing the motor similarity between the SMA Motor and Stepper Motor was used. The motor had been simulated and the results were verified experimentally. Various Phase Sequencing techniques

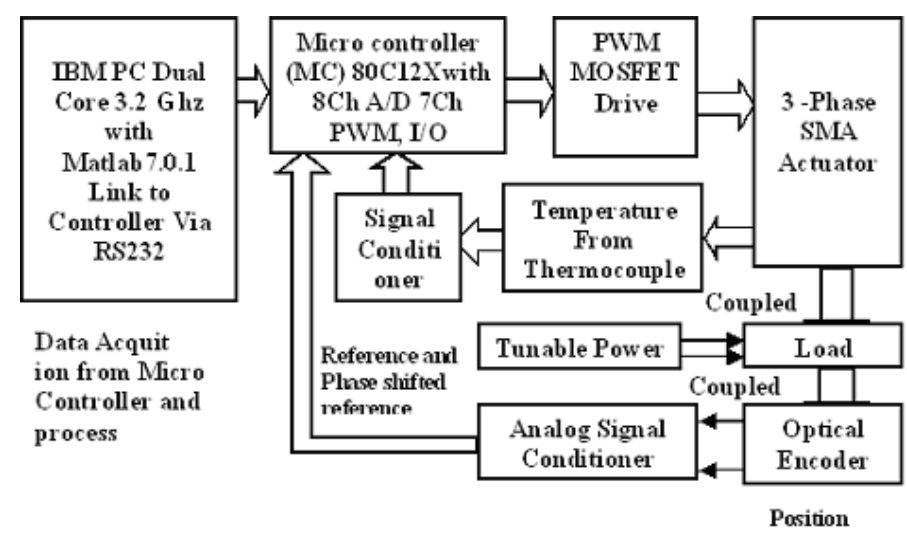

Figure 18. Schematic of electronic hardware for sequencing. 


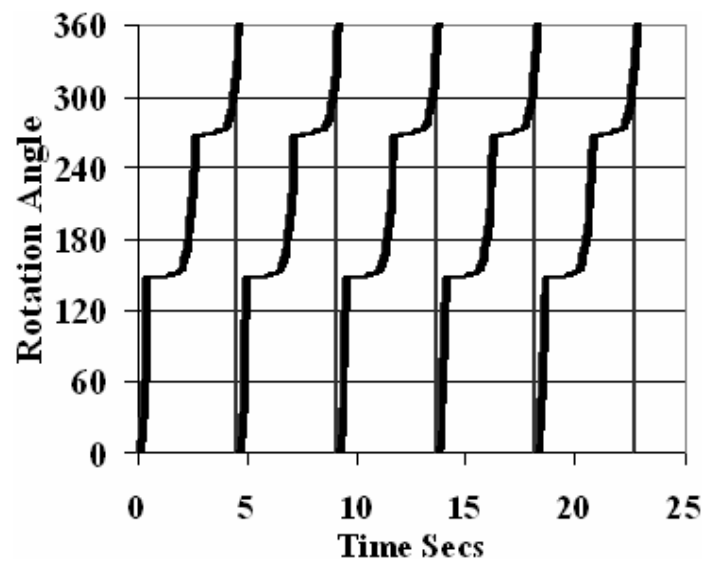

Figure 19. Experimental phase sequencing of motor for continuous rotation.

for actuating the motor are presented and their advantages and limitations were discussed. While One Phase on Sequencing consumes less energy, Improved One Phase on Sequencing additionally provides better torque at the cost of feedback. The SMA Motor with phases above 5 can be controlled using Poly Phase Sequencing. Poly Phase Sequencing produces the maximum torque compared to the other methods of sequencing. The SMA Motor presented was found to be robust, smooth and silent during operation.

\section{References}

Dominiek Reynaerts, Hendrik Van Brussel 1998 Design aspects of shape memory actuators. Mechatronics 8(6): 635-656

Duerig T W, Melton K N, Stockel D, Wayman C M 1990 Engineering aspects of shape memory alloys (London: Butterworth) Heinemann, Part I \& Part II

Kuo B C 1979 Incremental Motion Control, Step Motors and Control Systems, Vol. II, SRL Publishing Co, $1-90$

Kuribayashi K 1989a A new servo motor using shape memory alloy. Industrial Electronics Society, IECON'89 15th Annual Conference of IEEE, 1: 238-243

Kuribayashi K 1989b Millimeter size joint actuator using shape memory alloy. Industrial Electronics Society, IECON'89, 15th Annual Conference of IEEE, 1: 139-144

Mellor B G 1989 Engineering properties of shape memory alloys. In: J M Guilemany (Ed.), Science and technology of Shape Memory Alloys Palma de Mallorca: Univ. Illes Balears 335-395

Otsuka K, Wayman C M 1989 Shape memory materials, Cambridge University Press, Chapter 11 